\title{
Treatment of reflux strictures of the oesophagus by the Nissen-Rossetti operation
}

\author{
G. KENT HARRISON and B. M. GOMPELS \\ St. Thomas' Hospital, London
}

\begin{abstract}
Eight patients with bougie-resistant strictures of the oesophagus due to gastric reflux have been treated by the Nissen-Rossetti operation. Follow-up clinical and barium studies show the results to be encouraging. The principle involved is the prevention of reflux.
\end{abstract}

There are several forms of treatment available for a stricture of the oesophagus caused by reflux of gastric fluid. In this context only strictures which resist the passage of bougies and the oesophagoscope are included. A constant narrowing is shown on barium swallow. If sufficient barium passes into the stomach a hiatus hernia and reflux are seen. The stricture is in the region of the junction of squamous mucous membrane and the non-acidsecreting fundal type of gastric mucous membrane. As this latter type of mucous membrane may line the lower oesophagus, the stricture may be some distance above the herniated stomach (Barrett, 1950).

Repeated dilatations have enabled many patients to carry on with a fluid diet, but the stricture very often becomes progressively tighter and perforation by bougie is a significant risk.

Collis (1965) managed 69 patients with benign oesophageal strictures by gastroplasty. $\mathrm{He}$ believed that the stricture could be controlled by preventing the reflux.

Belsey (1966) reported his experience with colon transplants. He achieved an $81 \%$ success rate. In his operation the stomach was wrapped around the lower end of the transplanted colon to prevent refiux from the stomach to the colon (Belsey, 1965). He found that oesophagogastrostomy was unsatisfactory, with a success rate of $44 \%$.

Brain (1967) described his experience with jejunal transplantation for simple strictures of the oesophagus. Relief of all symptoms was obtained in $73 \%$ of the survivors. Post-operative barium reflux was noted in $72 \%$.

Barrett (1962) observed that in resected specimens of the strictured area, the whole length of the palpable mass was composed of submucous fibrosis on the inside and hypertrophied muscle on the outside. Some resolution is possible in each of the elements causing the obstruction.

Nissen and Rossetti (1963) described the prevention of gastric reflux by enfolding the lower oesophagus with the fundus of the stomach.

In the operation used in these patients the left chest is opened through the eighth interspace. A lateral incision is made in the diaphragm (Harrison and Norton, 1969). The oesophagus is freed to the level of the hilum with preservation of the lower oesophageal artery. The oesophagus and stomach are freed from the hiatus. The lesser curvature is freed to below the left gastric artery and the greater curvature for about half its length. After this a large part of the stomach can be

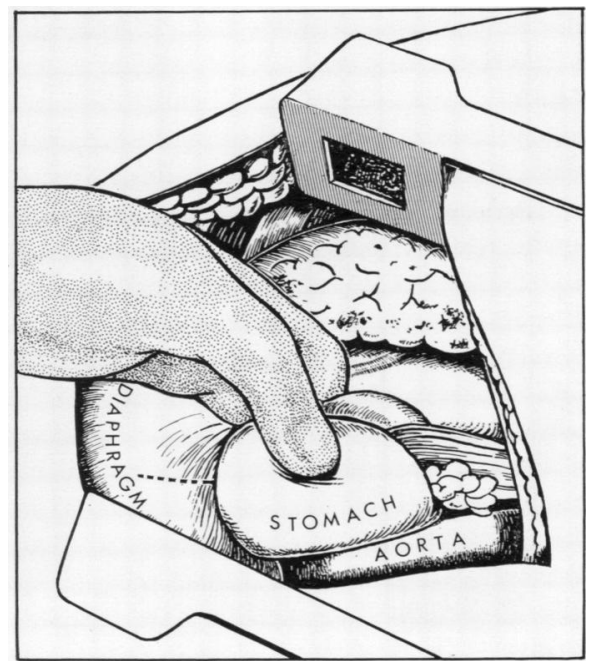

FIG. 1. The stomach surrounds the lower oesophagus preparatory to stitching. Dotted line shows site of the radial incision in diaphragm. 
elevated through the hiatus without tension. It is laid postero-lateral to the oesophagus and used to enfold the lower 2-3 in. $(50-76 \mathrm{~mm}$.) of the oesophagus. This includes most of the thickened oesophagus in strictures of the lower end (Fig. 1). The stomach is sutured to the oesophagus in a transverse plane at the superior border of the encirclement, and the two vertical enfolding edges of stomach are sutured to the oesophagus and to each other. A size $24 \mathrm{~F}$ or larger tube or bougie and a nasogastric tube are rassed into the stomach before the enfolding. The large tube is removed after this. Fingers around the oesophagus in the chest help to pass the bougie.

A few sutures are placed between the stomach and the antero-lateral hiatus edge. Posteriorly, the hiatus is left open and stitches are placed from the stomach to any areolar tissue available. Recently, the hiatus has been enlarged by a radial incision 1 in. $(25.4 \mathrm{~mm}$.) in length, as there is some evidence from other patients that with a gain in weight gastric obstruction can develop at the hiatus.

The patient who required resection had had a pyloroplasty at a previous operation. If a pyloroplasty had been required at the time of resection the chest would have been closed and an abdominal incision made for the approach.

\section{CASE REPORTS}

CASE 1 L.M., a woman aged 66, complained of heartburn for 10 years and dysphagia for five years. A

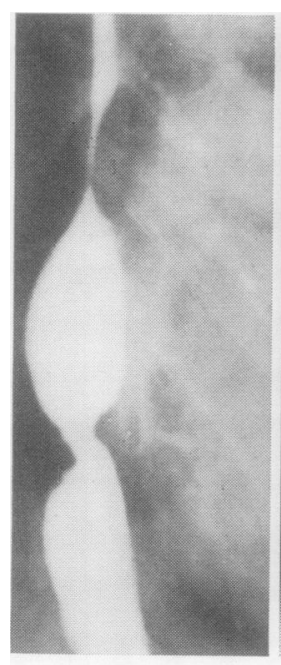

(a)

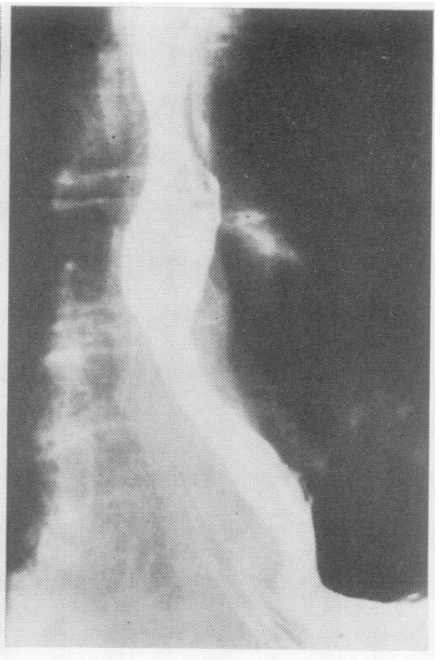

(b)
FIG. 2. Case 1. (a) Oesophageal stricture 3 in. (76 mm.) above the hiatus. (b) Complete disappearance of the stricture four years following surgery. barium meal in December 1965 (Fig. 2a) showed ass stricture 3 in. ( $76 \mathrm{~mm}$.) above the hiatus with an intero vening hiatus hernia and reflux. A Nissen-Rossetti operation was performed in February 1966. The oeso $\overline{\bar{\eta}}$ phagus was much thickened in the strictured area. $A_{\bar{D}}^{-}$ 24F bougie was passed with difficulty. Since operation there have been no symptoms of reflux and swallow ing has steadily improved to become normal. A four $\vec{P}$ year radiological follow-up on 4 February $1970^{\circ}$ (Fig. 2b) showed that the stricture had completely $\vec{\omega}$ resolved and there was no hold up of barium oros evidence of reflux.

CASE 2 A.M., a woman aged 51, complained of progressive dysphagia for three years. There was no clear. history of reflux. A barium meal in January 1966 showed a stricture of the lower oesophagus with theo appearance of a penetrating ulcer. A hiatus hernia and ${ }^{\supset}$. reflux were present. In March 1966 a Nissen-Rossettic operation was performed. Considerable peri-oeso phageal adhesion was present and the oesophagus was very thickened in its lower 2 in. $(50 \cdot 8 \mathrm{~mm}$.). Dilatation was difficult. Within three weeks the patient swallowed soft solids. Since operation there have been two daylong episodes of dysphagia which resolved spon-taneously. Otherwise she has eaten normally. Barium examination on 16 April 1970, four years postoperatively, showed neither stricture nor hold up. Very slight reflux of barium was observed.

CASE 3 R.W., a boy aged 16, had had symptoms of reflux since the age of 3 , with previous operations at $\overrightarrow{\bar{\sigma}}$ the ages of 3 and 14 years. However, the symptoms 3 of dysphagia and reflux persisted. He frequently twisted his head to the left, contracted his abdominal muscles and regurgitated bitter fluid. This relieved a feeling of discomfort in the abdomen. A barium meal in June 1967 showed a stricture just below the level of the aortic arch. A hiatus hernia and reflux were present. There were $3-4$ in. (76-102 mm.) of oeso- $\frac{\sigma}{3}$ phagus between the stomach and the stricture. Oesophagoscopy showed an ulcerated narrow area ato $35 \mathrm{~cm}$. This obstructed the oesophagoscope but a $24 \mathrm{~F}$ bougie was eased through it. Biopsies showedo squamous epithelium proximal and fundal type mucosa distal to the stricture. In July 1967 a Nissen-o Rossetti operation was performed. The oesophagus was thickened and surrounded by much fibrosis. Only ${ }_{\sigma}$ at the level of the aortic arch did the oesophagus feel normal. A bougie was passed with considerable difficulty through the stricture 4 in. $(102 \mathrm{~mm}$.) above the diaphragm. The lower 3 in. (76 mm.) of the oesophagus were enfolded, leaving the stricture area free Since operation he has swallowed normally with no symptoms of reflux. The movements of the head previously associated with reflux have stopped. Barium examination performed on 10 April 1970, almost three years post-operatively, showed a normal looking oeso- $\frac{}{\mathbb{D}}$ phagus with no evidence of stricture, hold up or reflux. $\frac{\rho}{\square}$

CASE 4 I.G., a man aged 63, had symptoms of heartburn for many years with dysphagia for five months. 
In September 1966 a barium meal showed a low oesophageal stricture with hiatus hernia and reflux. In February 1967 a Nissen-Rossetti operation was performed. The oesophagus was firm and thick for 1 in. $(25 \mathrm{~mm}$.) above the hernia. Considerable pressure was required to pass a dilating bougie through this area. This patient has not had a satisfactory result and the reason is not clear. Dysphagia persists after dilatation on two separate occasions. A stricture and excess oedematous mucous membrane were found but no ulceration. Repeated barium examinations showed a persisting stricture with a variable degree of hold up. Reflux could not be excluded because the barium emptied rapidly into the duodenum. The stomach continues to surround the lower oesophagus. Dilatation with a Negus bag in April 1970 produced improvement.

CASE 5 P.H., a man aged 68, had symptoms of heartburn for one year with dysphagia for six months. A barium meal in January 1968 (Fig. 3a, b) showed a stricture of the lower oesophagus with hiatus hernia and reflux. In June 1968 a Nissen-Rossetti operation was performed. There was much thickening in and around the lower oesophagus and moderate resistance to the passage of a bougie. Dysphagia persisted but with proper dentures swallowing steadily improved. Considerable improvement was seen in a barium examination two months post-operatively (Fig. 3c). On 21 January 1970 , some 18 months post-operatively (Fig. 3d), neither hold up nor reflux was demonstrated.

CASE 6 O.M., a woman aged 67, had reflux symptoms for seven years with severe dysphagia for one year, producing loss of $3 \mathrm{st}$. (19 kg.) in weight in three years. In September 1967 vagotomy and pyloroplasty were performed without relief. In March 1968 a barium meal showed stricture of the lower oesophagus with hiatus hernia and reflux. At operation in September 1968 a leiomyoma was found in the strictured area. Bougies could not be passed. With a finger in the stomach no opening could be felt into the oesophagus. The lower oesophagus disintegrated with manipulation. A local resection of the lower end of the oesophagus and adjacent stomach with re-establishment of the continuity of the gut by an oesophagogastrostomy was performed. One inch $(25 \mathrm{~mm}$.) of oesophagus and the end to side anastomosis were enfolded by stomach. Swallowing has steadily improved to normal, except for an occasional shortlasting sensation of hold up. A barium meal on 15 April 1970, 19 months post-operatively, showed narrowing at the anastomosis. There was minimal hold up and reflux was not present.

CASE 7 L.W., a woman aged 76, had symptoms of reflux and increasing dysphagia for four years. In December 1968 a barium meal showed a stricture in the lower oesophagus with hiatus hernia and reflux. In January 1969 a Nissen-Rossetti operation was performed. The lower oesophagus was thickened and there was moderate resistance to the passage of a bougie. The post-operative course was complicated and death occurred from a massive pulmonary embolus.

CASE 8 E.T., a woman aged 57, had symptoms of gastric reflux for 25 years and dysphagia for 18 months, during which time she had three oesophageal dilatations. At the fourth oesophagoscopy in

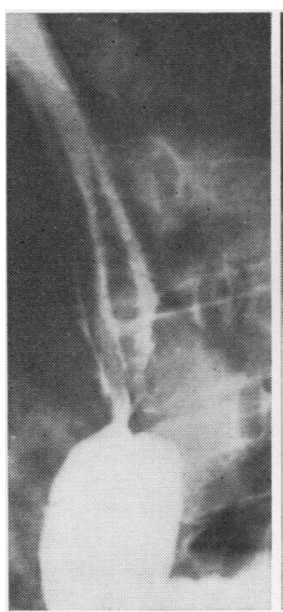

(a)

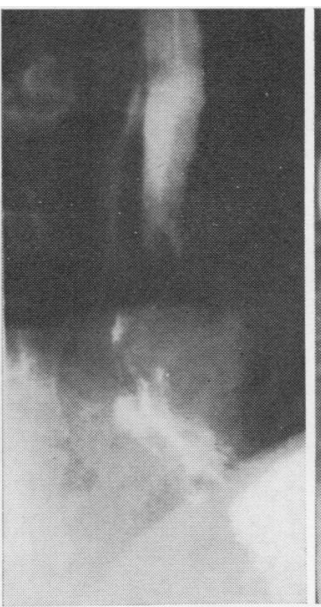

(b

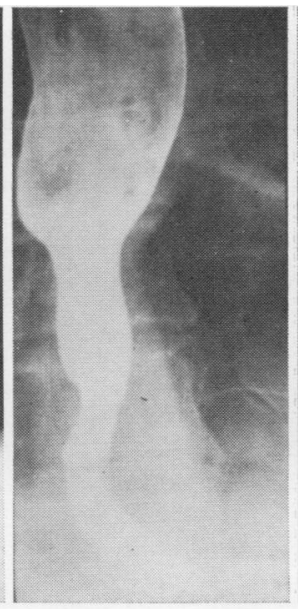

(c)

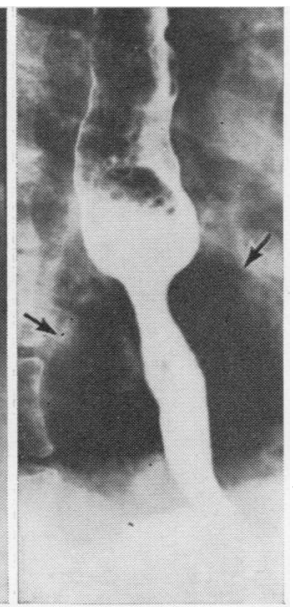

(d)

FIG. 3. Case 5. (a, b) Stricture of the lower oesophagus with hiatus hernia and reflux.

(c) Considerable improvement two months after surgery. (d) No stricture is seen. Gas in the stomach surrounding the oesophagus is clearly demonstrated (arrowed). 
November 1969 it was not possible to pass a bougie. A barium meal in May 1968 showed a stricture with an ulcer about 2 in. (50 mm.) above the hiatus, with hiatus hernia and reflux. In November 1969 a NissenRossetti operation was performed. This had been delayed because of gross bilateral bronchiectasis and general frailty. The lower 4 in. $(102 \mathrm{~mm}$.) of oesophagus were thick, firm and adherent to surrounding tissues. The stricture was dilated by a finger forcibly pushed through from an incision in the fundus. Since operation swallowing has steadily improved. There are no symptoms of reflux. A barium meal performed three weeks post-operatively showed that the ulcer had disappeared. Examination in April 1970, five months post-operatively, showed further improvement. Neither reflux nor sıgnificant hold up was seen.

\section{DISCUSSION}

In the seven cases of conservative operation described, there have been five successes, one relative failure and one death. The single case of resection and enfolding is successful. The longest and shortest follow-ups have been four years and six months respectively. The improving quality of life in the successful cases has been dramatic.

In these patients it was not possible to reduce the oesophagus into the abdomen without undue tension or without elevating the diaphragm. The fate of the vagus nerves is not clear. In all cases they must have been distorted; in some they were not seen beside the stricture. Gastric secretion tests have not been done, but there is no evidence of pyloric obstruction.

This operation prevents reflux, apparently by the embracing action of the inner wall of the stomach on the oesophagus and by the transmission of abdominal pressure in the lumen of the stomach around the oesophagus. Pearson, Henderson, and Parrish (1969) showed a well-defined pressure change between the oesophagus and the $\varrho$ stomach following oesophagogastrostomy with o inkwell invagination of the oesophagus into the $\vec{\circ}$ stomach.

The evidence from these cases suggests that, if $\vec{\omega}$ the stricture can be enlarged to accept a 24 to $26 \mathrm{~F}$ bougie, it is worth trying this conservative operation. A resection with enfolding of the oesophagus $\widetilde{N}$ could be done at a later date if the operation. described fails.

Our thanks are due to the physicians and surgeons who referred their patients ; to Miss C. J. Dewe of the photographic department; and to Dr. R. Corbett.

\section{REFERENCES}

Barrett, N. R. (1950). Chronic peptic ulcer of the oesophagus and oesophagitis. Brit. J. Surg., 38, 175.

(1962). Benign stricture in the lower esophagus. J. thorac. cardiovasc. Surg., 43, 703.

Belsey, R. (1965). Reconstruction of the esophagus with left colon. J. thorac. cardiovasc. Surg., 49, 33.

(1966). Benign strictures of the oesophagus. Proc. roy Soc. Med., 59, 932.

Brain, R. H. F. (1967). The place for jejunal transplantation in the treatment of simple strictures of the oesophagus. Ann. roy. Coll. Surg. Engl., 40, 100.

Collis, J. Leigh (1965). Symposium on peptic stricture of the oesophagus. Thorax, $20,288$.

Harrison, G. K., and Norton, R. (1969). Prevention of reflux from the stomach into the oesophagus. Thorax, 24, 595.

Nissen, R., and Rossetti, M. (1963). Surgery of the cardia ventriculi. Ciba Symposium, 11, 195.

Pearson, F. G., Henderson, R. D., and Parrish, R. M. (1969). AnO operative technique for the control of reflux following esophagogastrostomy. J. thorac. cardiovasc. Surg., 58, 668. 